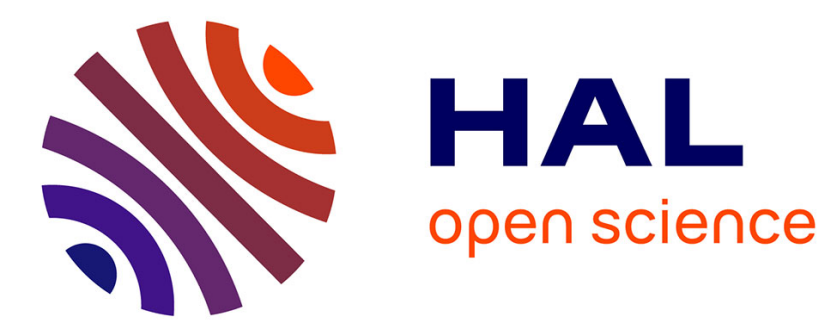

\title{
Procathepsin L secretion, which triggers tumor progression, is regulated by Rab4A in human melanoma cells
}

Alice Barbarin, Raymond Frade

\section{- To cite this version:}

Alice Barbarin, Raymond Frade. Procathepsin L secretion, which triggers tumor progression, is regulated by Rab4A in human melanoma cells. Biochemical Journal, 2011, 437 (1), pp.97-107. 10.1042/BJ20110361 . hal-00600761

\section{HAL Id: hal-00600761 https://hal.science/hal-00600761}

Submitted on 16 Jun 2011

HAL is a multi-disciplinary open access archive for the deposit and dissemination of scientific research documents, whether they are published or not. The documents may come from teaching and research institutions in France or abroad, or from public or private research centers.
L'archive ouverte pluridisciplinaire HAL, est destinée au dépôt et à la diffusion de documents scientifiques de niveau recherche, publiés ou non, émanant des établissements d'enseignement et de recherche français ou étrangers, des laboratoires publics ou privés. 
PROCATHEPSIN L SECRETION, WHICH TRIGGERS TUMOR PROGRESSION, IS REGULATED BY RAB4A IN HUMAN MELANOMA CELLS

\author{
Alice BARBARIN and Raymond $\operatorname{FRADE}^{(1)}$ \\ INSERM U.672 (former U.354), Immunochemistry of Cell Regulations and Virus Interactions, \\ Bâtiment G8, 5, rue Henri Desbruères, 91030 - EVRY Cedex, FRANCE \\ (1) Correspondence to: \\ Pr. Raymond FRADE, \\ Translational Research on Melanoma and Cancer, Université Versailles Saint-Quentin, Hôpital \\ Ambroise-Paré, 9, avenue Charles de Gaulle, 92104 - Boulogne Billancourt, Cedex, FRANCE. \\ Tél: 331490946 02; mail: raymond.frade@inserm.fr
}

Running Title: Rab4A regulates procathepsin L secretion 


\begin{abstract}
The switch of human melanoma cell phenotype from non to highly tumorigenic and metastatic is triggered by the increase of procathepsin $\mathrm{L}$ secretion which modifies tumor microenvironment. Our aim was to identify components involved in the regulation of procathepsin $\mathrm{L}$ secretion in melanoma cells. We focused on Rab family members, i.e. Rab3A, Rab4A, Rab4B, Rab5A, Rab8A, Rab11A, Rab27A and Rab33A, involved in distinct regulatory pathways. From analysis of mRNA and protein expression of these Rab components and their knock down by specific siRNAs emerged that Rab4A protein is involved in the regulation of procathepsin $\mathrm{L}$ secretion. This result was strengthened as procathepsin $\mathrm{L}$ secretion was either inhibited by expression of Rab4A dominant negative mutant or increased by overexpression of the Rab4A wild type Rab4A regulation: 1) discriminates between procathepsin $\mathrm{L}$ secretion and expression of intracellular cathepsin L forms; 2) did not modify other Rab proteins and GAPDH expression or IL-8 and MMP2 secretion; 3) still efficient on unglycosylated procathepsin L secretion. Thus, down- or up-regulation of Rab4A expression or Rab4A function triggered inhibition or increase of procathepsin $\mathrm{L}$ secretion, respectively. Furthermore, Rab4A regulation, by modifying procathepsin $\mathrm{L}$ secretion, switches the tumorigenic phenotype of human melanoma cells in nude mice.
\end{abstract}

Key words: Procathepsin L Secretion, Rab4A, Knock down, Melanoma, Metastatic, Nude Mice. 


\section{Introduction}

The switch of human melanoma phenotype from non to highly tumorigenic and strongly metastatic is under the control of intracellular and extracellular molecular events which contribute to modify the tumor microenvironment [1]. Among these, we undertook to identify proteinases involved in the resistance of melanoma cells to complement-mediated cell lysis and we focused on the identification of proteinases that cleave human $\mathrm{C} 3$, the third component of complement [2]. Indeed, cleavage of C3 inhibits its deposition on the cell surface and consequently complementmediated cell lysis. In addition, C3 cleavage generates C3 fragments involved in many biological functions, through at least, six distinct $\mathrm{C} 3$ receptors [3-5].

This led us to demonstrate that highly tumorigenic and strongly metastatic human melanoma cells oversecrete procathepsin L, a cysteine proteinase, which cleaves human C3 and contributes to cell resistance to complement-mediated cell lysis $[6,7]$. Procathepsin $L$ is the proenzyme of cathepsin L, a papain-type proteinase [8-10]. In normal cells, cathepsin L is stored in lysosomes as cathepsin L mature forms, while in tumor cell secretion of procathepsin L is strongly increased [2, 11-13]. Thus, cathepsin $\mathrm{L}$ acts as an endopeptidase, which degrades a wide range of intracellular cytoplasmic and nuclear proteins [10] and when secreted under procathepsin L form, also extracellular proteins $[2,7]$.

Four complementary results supported that the increase of procathepsin $\mathrm{L}$ secretion is a major event involved in the switch of human melanoma phenotype to highly tumorigenic and strongly metastatic. First, inhibition of secreted cathepsin L activity by polyclonal anti-cathepsin L (CTSL) antibodies $(\mathrm{Ab})$, prevented tumor formation and decreased by $50 \%$ metastasis development in lungs of nude mice [6]. Second, the increase of procathepsin L secretion in generated clones up-regulated C3-cleaving activity and switched their phenotype to highly tumorigenic and strongly metastatic [7]. Third, after designing an anti-human CTSL single chain variable fragment (ScFv) [14], we demonstrated that its stable transfection in high tumorigenic and strong metastatic human melanoma cells, triggered inhibition of procathepsin L secretion and consequently switched their phenotype to poorly tumorigenic and non metastatic [15]. Fourth, we demonstrated that injection of a lentiviral construct carrying this anti-CTSL-ScFv into tumors already induced in nude mice by human melanoma cells inhibits tumor progression [16].

From all these data, our aim was herein to analyze whether the Rab (Ras-like proteins in brain) proteins might be involved in procathepsin L secretion by melanoma cells. Indeed, Rab GTPase proteins are major regulators of the intracellular vesicular transport between different organelles of the endocytic and secretory pathways [17]. Rab proteins are involved in all steps of vesicular transport as vesicle formation, budding from the donor compartment, transport to the acceptor compartment, vesicle fusion and release of the vesicle content into the acceptor compartment. Rab proteins are particularly localized into specific intracellular compartments consistent with their involvement in distinct vesicular transport processes [17]. Cathepsin $\mathrm{L}$ is successively translated as preprocathepsin L, transferred through the Golgi apparatus as procathepsin L, then stored in lysosomes as cathepsin L mature forms [18]. Thus, we focused on major Rab proteins, i.e. Rab3A, Rab8A and Rab27A, involved in the secretion from the Golgi apparatus [19, 20]; Rab4A, Rab5A and Rab11A, involved in the transport between the endosomal compartment and the plasma membrane [21] and Rab33A, down-regulated in malignant melanoma [22].

Thus, we used herein various methods to analyze the putative involvement of these Rab family members in procathepsin L secretion. First, we analyzed their expression in four human melanoma cell lines, characterized by different level of procathepsin L secretion and consequently by distinct tumorigenic and metastatic phenotypes, as already demonstrated [7, 15, 16, 23, 24]. Second, in order to discriminate between these Rab proteins, we transfected melanoma cells with specific small interfering RNAs (siRNAs). From these two first steps, only Rab4A emerged as involved in procathepsin L secretion. Expression level and pattern of intracellular cathepsin L forms were not 
significantly modified by Rab4A expression. Third, these two first results were strengthened by stable transfections of melanoma cells with either a Rab4A dominant negative mutant form to inhibit endogenous Rab4A function or the Rab4A wild type form to over-express Rab4A. Fourth, modification of Rab4A function or Rab4A expression, by regulating procathepsin $\mathrm{L}$ secretion switched the progression of tumors induced by human melanoma in nude mice. All together, these data clearly demonstrate that Rab4A expression and Rab4A function regulate procathepsin $\mathrm{L}$ secretion in human melanoma cells. 


\section{Materials and Methods}

\section{Cell lines and culture conditions}

Four human melanoma cell lines, derived from four patients and kindly provided by Dr M. BarEli (M. D. Anderson Cancer Center, Houston, TX, U.S.A.) [25] were used and already tested: highly tumorigenic and strongly metastatic A375SM [15, 16, 24] and DM-4 [6] cells and non/poorly tumorigenic and non-metastatic DX-3 [7, 23] and SB-2 [24] cells. The cells were grown as previously described [23]. When needed, tunicamycin (Sigma), dissolved in DMSO, was added to cell cultures at a final concentration of $10 \mu \mathrm{g} / \mathrm{ml}$ for $24 \mathrm{~h}$.

\section{Plasmid construction}

Human Rab4A cDNA was amplified from A375SM cells by using 5' primers flanked with the underlined EcoRI restriction site (5'-GGAATTCGatgtcgcagacggccatgtc-3') and 3' primers flanked with the underlined HindIII restriction site (5'-agtggcatttgggacacaatcgAAGCTTCAC-3') and was subcloned in the pCMV-Tag3A plasmid (Stratagene) in frame with DNA encoding a N-terminal myc epitope. The mutant construct Rab4AS22N was obtained by site directed mutagenesis (QuickChange mutagenesis kit; Stratagene) of the wild type Rab4A plasmid, using the primer 5'GCAGGAACTGGCAAAAATTGCTTACTTCATCAG-3' containing underlined mutated nucleotides. Sequences of the wild type and mutated Rab4A expression vectors were verified by sequencing (Cogenics).

\section{Stable transfection of melanoma cells}

A375SM or DX-3 melanoma cells were seeded in $60 \mathrm{~mm}$ tissue culture plates, cultured for $24 \mathrm{~h}$, and transfected by using $8 \mu \mathrm{l}$ of JetPEI (Polyplus Transfection) and $4 \mu \mathrm{g}$ of pCMV-Tag3A empty control vector or pCMV-Tag3A-myc-Rab4A or pCMV-Tag3A-myc-Rab4AS22N expression vectors, as indicated in each set of experiments. Sixteen hours after transfection, the medium was changed to complete culture medium. Selection was performed after $48 \mathrm{~h}$ with culture medium containing G418 at $1.2 \mathrm{mg} / \mathrm{ml}$ and G418-resistant cells were established in culture.

\section{RNA interference}

Small interfering RNAs (siRNAs) were purchased from Invitrogen (Stealth ${ }^{\mathrm{TM}}$ RNAi). The sequences of siRNAs were 5'-GCAACGACAAGAGGAUCAAGCUGCA-3' for Rab3A, 5'A A C C A A C A G A A UCA GUGUUGUAG-3' for Rab5 A, $5^{\prime}$ ACAAGAGACAAGUUUCCAAGGAACG-3' for Rab8A, and 5'CAAUAAGAgUGAUCUACGUCAUCUC-3' for Rab11A. The Rab4A knock down was performed with two specific siRNAs alone or in combination: 5' ACGGCCAUGUCCGAAACCUACGAUU-3' (Rab4A siRNA1) and 5'GAUGCAGAUCGUGAAGUUACCUUCU-3' (Rab4A siRNA2). Two negative control siRNAs (medium GC and low GC Stealth ${ }^{\mathrm{TM}}$ RNAi; Invitrogen) were used depending on the GC content of the corresponding Rab siRNA used. Scrambled Rab4A siRNA 1 5'GCGGAACGCUAUGCCCAUUACACUA-3' was also used as a control for Rab4A inhibition. Transfections were performed in 12-well plates using the Interferin reagent (PolyPlus Transfection). siRNAs were transfected at a final concentration of $5 \mathrm{nM}$ except for Rab8A, at $10 \mathrm{nM}$, and for combination of two siRNAs, each at $2.5 \mathrm{nM}$. Analysis of mRNA expression or protein expression and secretion were performed $72 \mathrm{~h}$ after transfection.

\section{Reverse transcription PCR}

Expression of mRNA was evaluated by semi-quantitative or quantitative reverse transcriptionPCRs (RT-PCRs). Briefly, the total RNA was isolated from melanoma cells using TRIzol reagent (Invitrogen) and then reverse transcribed. For semi-quantitative analysis, the PCR reaction was carried out with 24 amplification cycles for glyceraldehyde-3-phosphate dehydrogenase (GAPDH) 
and cathepsin L using specific primers [15], 23 amplification cycles for Rab11A using primers 5'AGCAAGAGCACCATTGGAGT-3' and 5'-TGGCTTGTTTTCAGTGGTTG-3', 32 amplification cycles for Rab5A using primers 5'-CTTCAGAGGCAAGCAAGTCC-3' and 5'TTGTGTGGGTTCGGTAAGGT-3' and 29 amplification cycles for Rab3A, Rab4A and Rab8A using primers 5'-TACCGGACCATCACCACCGCATAC-3' and 5'CAGATGACATCCACCAGGCGCTCAAA-3' for Rab3A; 5'-CCATGTCCGAAACCTACGAT-3' and 5'-ATGTTCTGGCTCGCTAGCAT-3' for Rab4A and 5'-GGAACTGGATTCGCAACATT-3' and 5'-TTTTGACTCCCTGGTTGCTC-3' for Rab8A. PCR products were then analyzed on a $1.5 \%$ agarose gel containing ethidium bromide. For quantitative analysis, Real-Time RT-PCR was performed by Beckman Coulter Genomics (Meylan, France) with an Applied Biosystems 7500 Fast Real-Time RT-PCR System using SYBR Green. Cathepsin L amplification primers were 5'TGTGGGGCCCATTTCTGTTGCTA-3' and 5'- CATGCGCCATCCCCAGTCAAGT-3'. The amount of cathepsin L transcript in each RNA sample was quantified and normalized to GAPDH content. Relative amounts of cathepsin L cDNA were calculated by the relative comparative $2-\Delta \Delta \mathrm{Ct}$ method and are expressed as a percentage of cathepsin L cDNA measured in non-transfected cells.

\section{Cathepsin L activity}

Cathepsin L activity was determined using the fluorogenic synthetic substrate Z-Phe-Arg-AMC (Calbiochem). Assays were performed at $37^{\circ} \mathrm{C}$ using $\mathrm{A} 375 \mathrm{SM}$ cell extracts or conditionned extracellular media in a sodium acetate buffer $(\mathrm{pH}$ 5) containing EDTA, DTT and CA-074 ([L-3trans-(Propylcarbamoyl)oxirane-2-carbonyl]-L-isoleucyl-L-proline), the specific cathepsin B Inhibitor III (Sigma Aldrich), to rule out any interference with cathepsin B activity. In addition, in the assay reported (Figure 6C, lane 4), the specific inhibitor Z-Phe-Phe-CHN2 of cathepsin L (Calbiochem) was added. Fluorescence measurements were carried out on a multiplate reader Victor 2 Wallac (Perkin-Elmer) and relative fluorescent units (RFU) were measured at $460 \mathrm{~nm}$ using an excitation wavelength of $355 \mathrm{~nm}$.

\section{Cellular extract and extracellular media preparation and analysis}

Cells were lysed in PBS containing 1\% (v/v) Nonidet P-40 supplemented with a combination of proteinase inhibitors (Roche Diagnostics). Conditioned extracellular media were prepared by incubating cells in freshly serum-free culture medium for $24 \mathrm{~h} \mathrm{[7].} \mathrm{Both} \mathrm{cell} \mathrm{media} \mathrm{were} \mathrm{analyzed}$ by western blot using anti-cathepsin $\mathrm{L}$ moAb (clone 3D8) prepared in our laboratory [14], antiGAPDH moAb (moAb374, Chemicon International), anti-myc epitope moAb (clone 4G10, Upstate) anti-Rab3A Ab (Santa Cruz Biotechnologies), anti-Rab8A moAb (BD Transduction Laboratories), anti-Rab11 A Ab (Zymed Laboratories), anti-Rab4A Ab and anti-Rab5A Ab (Stressgen, Assay Design). Bound antibodies were detected with peroxidase-linked immunoglobulin $\mathrm{Ab}$ (Dako) and the enhanced chemiluminescence system (Amersham Biosciences). Secreted interleukin-8 (IL-8) was measured in conditioned media by using the ELISA MAX ${ }^{\mathrm{TM}}$ set deluxe kit for human IL-8 (Biolegend), according to the manufacturer's instructions. Secreted MMP-2 (Matrix Metallo Proteinase 2) protein was measured in conditioned media by western blot using anti-MMP-2 moAb (Ab-3, Calbiochem) and quantified, as previously described [23]. Indeed, we verified that a good correlation existed in our samples between the detection of MMP-2 protein in samples by anti-MMP-2 moAb and the measure of MMP-2 activity by zymography gel. Thus, we herein report the level of secreted MMP-2 protein. The intensity of each band of western blots was quantified by using the ImageJ64 freeware (W. S. Rasband, National Institutes of Health, Bethesda, MD; http://rsb.info.nih.gov/ij/), then compared to those obtained with cells transfected with control siRNA, the best control and expressed as relative band intensity (r.b. i.). 


\section{Pulse-Chase Analysis}

A375SM melanoma cells were starved for $60 \mathrm{~min}$ at $37^{\circ} \mathrm{C}$ in $5 \mathrm{ml}$ of methionine/cysteine-free Dulbeco's modified Eagle's medium (DMEM) (Invitrogen Life Technologies, Cergy Pontoise), supplemented with $2 \mathrm{mM}$ glutamine and $10 \%$ heat-inactivated FCS. Then, cells were incubated in 1 $\mathrm{ml}$ and pulse with $0.5 \mathrm{mCi} \mathrm{ml}-1$ of a mix of ${ }^{35} \mathrm{~S}$-methionine $/ 35 \mathrm{~S}$-cysteine (PerkinElmer, Courtaboeuf, France), for $60 \mathrm{~min}$ and chase in complete RPMI 1640 containing 5\% calf serum for $0,1,4,8$ and 16 hours. After each chase-point, cells were lysed in NP-40 lysis buffer, pH 7.4 (50 $\mathrm{mM}$ Tris, $150 \mathrm{mM} \mathrm{NaCl}, 0.5 \%$ NP-40) supplemented with $1 \%$ SDS, boiled for 5 min, then reconstituted to $1.5 \mathrm{ml} \mathrm{SDS}$-free NP-40 solution. The lysates were pre-cleared on irrelevant antibodies covalent-linked on beads and then submitted to immunoprecipitation using $20 \mu l$ of cathepsin L polyclonal antibodies covalent-linked on beads. Then, immunobeads were extensively washed at least 5 times, with $2 \%$ FCS in the first wash and immunoprecipitated compounds were analyzed under reducing conditions by SDS-PAGE .

\section{In Vivo tumor progression}

First of all, parental A375SM cells, characterized by a high-tumorigenic phenotype, were stably transfected with the Rab4AS22N expression vector with the Rab4A dominant negative mutant form (A375SM myc-Rab4AS22N) or with the empty vector (A375SM mock), used as control. In parallel, parental DX-3 cells, characterized by a non-tumorigenic phenotype, were stably transfected with the expression vector encoding for Rab4A wild type form fused also with the myc epitope or with the empty vector (DX-3 mock), used as control. Then, 7 × $10^{5}$ transfected cells, suspended in Hank's buffer in a $200 \mu 1$ volume, were injected subcutaneously (s.c.) into the right scapular region of nude mice. As previously described [16], the progression of the tumors was monitored by measuring these with calipers at the indicated days, as soon as the tumor volume of each mouse became detectable, using six mice per group. The volume of each tumor was calculated using the formula $\{($ length $\mathrm{x}$ width $\mathrm{x}$ height) $\mathrm{x}$ pi/6 (i.e. 0.52 ) $\}$. The data provided represent the mean value \pm SD calculated for each group. The mice were put to death 38 days after cell injection.

\section{Statistical analysis}

$\mathrm{P}$ values were calculated from the tumor volumes by using the two-tailed Student's t test and were considered to be significant and different from the control samples when the $\mathrm{P}$ value was less than 0.03 . 


\section{Results}

\section{Expression of Rab proteins in human melanoma cells}

The expression of Rab proteins was analyzed at the protein and the mRNA levels in four human melanoma cell lines characterized by different phenotypes: A375SM and DM-4 cells, highly tumorigenic and strongly metastatic phenotype related to high procathepsin L secretion, and DX-3 and SB2 cells, poorly or non-tumorigenic and non-metastatic phenotype related to low procathepsin L secretion $[2,7,15,16,23,24]$. The Rab proteins selected were Rab3A, Rab8A and Rab27A [19, 20], Rab4A and its isoform Rab4B [26], Rab5A and Rab11A [21] and Rab33A [22]. Rab protein expression was analyzed on total cell extracts by western blot using specific antibodies against each Rab protein (Figure 1). The quantification of western blots was performed as described in Materials and Methods. Data demonstrated that Rab4A protein was more expressed in A375SM and DM-4 cells than in DX-3 and SB-2 cells. Conversely, Rab5A expression was higher in DX-3 and SB-2 cells than in A375SM and DM-4 cells. In comparison, Rab8A was equally expressed in these four melanoma cells, while Rab3A and Rab11A expression was variable with no apparent correlation with the cell phenotype. The mRNA expression of these Rab family members was also analyzed in these four melanoma cell lines by semi-quantitative RT-PCR analysis (Figure 2). Data demonstrated that the pattern of Rab mRNA expression is similar to the profile of Rab protein expression for Rab3A, Rab4A, Rab8A and Rab11A, except for Rab5A. Indeed, the expression of Rab5A mRNA was similar in the four cell lines, a pattern different to Rab5A protein expression. Additional analysis also demonstrated that Rab4B, Rab27A and Rab33A mRNA and protein were not detected in these four human melanoma cell lines (data not shown). Thus, among the Rab proteins herein analyzed, only Rab4A expression correlates with the tumorigenic phenotype of these melanoma cell lines.

\section{Knock down of RabA4 expression decreases procathepsin $L$ secretion}

To analyze whether Rab proteins might be involved in procathepsin L secretion, we knocked down Rab protein expression in A375SM cells, which present the highest level of procathepsin L secretion $[15,16]$. Thus, parental cells (NT) were transfected with siRNAs specific for each Rab and then protein expression was analyzed in total cell extracts by western blot analysis, using specific anti-Rab Ab. Bands of each gel was quantified as described in Materials and Methods and compared to those obtained with cells transfected with non-targeting siRNAs (control siRNA) (lanes 2). As shown Figure 3, each specific siRNA induced effective inhibition of their respective Rab protein, i.e. more than $90 \%$ inhibition for Rab3A, Rab5A, Rab4A and Rab11A proteins (Parts A, B, C and E) and at least 60\% inhibition for Rab8A protein (Part D). Glyceraldehyde-3phosphate dehydrogenase (GAPDH) expression was not significantly modified thus used as control.

In the same set of experiments, we analyzed the level of intracellular expression of procathepsin $\mathrm{L}$ and cathepsin $\mathrm{L}$ mature forms and the level of procathepsin L secretion. Data demonstrated (Figure 3) that knock down of Rab3A (Part A), Rab5A (Part B), Rab8A (Part D) and Rab11A (Part E) in A375SM celis did not modify significantly the procathepsin L secretion in transfected cells. However, in transfected cells, Rab4A knock down by Rab4A siRNA1 (lane 3) or Rab4A siRNA2 (lane 4) induced a $51 \%$ and $63 \%$ decrease of procathepsin L secretion, respectively. In addition, in cells transfected with Rab4A siRNA1 and Rab4A siRNA2 together, i.e. Rab4A siRNAs(1+2), up to 80\% procathepsin L secretion was decreased (lane 5). An additional control was performed by analyzing the level of intracellular expression of Rab3A, Rab5A, Rab8A and Rab11A, in cells transfected with Rab4A siRNAs(1+2). Data demonstrated that Rab4A knock down by Rab4A siRNAs(1+2) did not modify significantly the expression of Rab3A, Rab5A, Rab8A and Rab11A proteins (Figure 4). 


\section{Regulation of procathepsin $L$ secretion by Rab4A expression}

From the above experiments (Figure 3C), emerged also that the total intracellular expression and the pattern of procathepsin L and cathepsin L mature forms were not significantly modified. This observation led us to analyze the time-course of cathepsin L biogenesis and maturation in A375SM melanoma cells transfected with Rab4A siRNAs $(1+2)$ or with control siRNA. For this purpose, pulse chase experiments were performed on transfected A375SM cells using a mix of $35 \mathrm{~S}$ methionine and ${ }^{35} \mathrm{~S}$-cysteine, as described in Materials and Methods. As shown Figure 5, the turnover of intracellular procathepsin L $(41 \mathrm{kDa})$ and two cathepsin L mature forms $(34 \mathrm{kDa}$ and 29 $\mathrm{kDa}$ ) was unchanged when A375SM cells were transfected with Rab4A siRNAs(1+2) (bands A), as compared to cells transfected with control siRNA (bands C). These results were also supported by semi-quantitative RT-PCR analysis (Figure 6A) and by quantitative Real-time RT-PCR analysis (Figure 6B). Both analysis demonstrate that cathepsin L mRNA expression was not modified in A375SM cells transfected with Rab4A siRNAs(1+2) (lane 3), as compared to cells transfected with control siRNA (lane 2). In these same assays, GAPDH mRNA expression was also not modified, giving an additional control (Figure 6A, lower panel). Furthermore, cathepsin L activity on the fluorogenic substrate Z-Phe-Arg-AMC was measured in cell extracts in which was also added the CA-074 methyl ester, a specific cathepsin B inhibitor to rule out any putative interference between the enzymatique activity of both proteinases. As shown Figure $6 \mathbf{C}$, cathepsin L activity was unchanged in cellular extracts prepared from cells transfected with Rab4A siRNAs(1+2) (lane 3), as compared to those prepared from cells transfected with control siRNA (lane 2). This activity was fully inhibited by the Z-Phe-Phe- $\mathrm{CHN}_{2}$, a specific inhibitor of cathepsin L [27] (lane 4). Cathepsin $\mathrm{L}$ activity on substrate Z-Phe-Arg-AMC was never detected in the extracellular conditioned media of melanoma cells, according to our previous data that only the proenzyme form was detected [27]. Thus, Rab4A down-expression inhibits procathepsin L secretion without significant modification of the turnover of the expression of intracellular cathepsin L forms and of cathepsin L activity.

In order to determine whether knock down of Rab4A expression might modify other secretion pathways, we analyzed also the amount of Interleukin-8 (IL-8) and Matrix MetalloProteinase 2 (MMP-2) secreted in extracellular medium of transfected A375SM cells. Indeed, IL-8 is a member of the CXC chemokine family. This chemokine, one of the major mediators of the inflammatory response, is a chemoattractant and also a potent angiogenic factor secreted by several cell types [28]. In addition, MMP-2, an important metallo-proteinase, is also secreted by human melanoma $[23,29]$. Secreted IL-8 was quantified by ELISA, as described in Material and Methods, while secreted MMP-2 was quantified by western blot as previously described [23]. As shown Figure 6D, knock down of Rab4A expression in A375SM cells transfected with Rab4A siRNAs(1+2) did not modify IL-8 secretion (black histogram) and MMP-2 secretion (white histogram) (lane 3), as compared to cells transfected with control siRNA (lane 2) or to non-transfected cells (lane 1). Thus, knock down of Rab4A expression did not regulated IL-8 or MMP-2 secretion.

To confirm the involvement of Rab4A in procathepsin $\mathrm{L}$ secretion, we used other methods, i.e. expression of Rab4A dominant negative mutant form that inhibits Rab4A function or overexpression of Rab4A. In a first set of experiments, we prepared the Rab4A dominant negative mutant form, Rab4AS22N, which is locked in the GDP-bound form and acts as a trans-dominant inhibitor of Rab4A vesicular transport [30]. This Rab4AS22N protein was fused to a myc epitope to allow its detection. Then, A375SM cells were stably transfected with the Rab4AS22N expression vector (A375SM myc-Rab4AS22N) or with the empty vector (A375SM mock), used as control. Rab4AS33N expression in transfected cells was determined by western blot analysis of total cell extracts using anti-myc epitope moAb. As shown Figure 7A, expression in transfected A375SM cells (lane 3) of Rab4AS22N protein, characterized by a $28 \mathrm{kDa}$ molecular weight slightly higher than the endogenous Rab4A, induced a $58 \%$ inhibition of procathepsin L secretion, as compared to cells transfected with the empty vector (lane 2). The expression and the pattern of intracellular cathepsin L forms (middle panel) were not significantly modified (lane 3 versus lane 2). Then, in a second set of experiments, cells were transfected with an expression vector encoding for Rab4A 
wild type form also fused with the myc epitope, i.e. myc-Rab4A. As shown Figure 7B, Rab4A overexpression in transfected A375SM cells induced a $\mathbf{6 4 \%}$ increase of procathepsin L secretion (lane 3, lower panel), as compared to cells transfected with the empty vector (lane 2), with no significant modification of the expression and the pattern of intracellular cathepsin L forms (middle panel). We also verified that Rab4A involvement in procathepsin $\mathrm{L}$ secretion was not restricted to A375SM cells. For this purpose, we used DM-4 melanoma cell line also characterized by a high level of procathepsin L secretion [2, 6]. As shown Figure 7C, Rab4A knock down (upper panel) in DM-4 cells inhibits with efficiency up to $80 \%$ procathepsin L secretion (lower panel). In addition, we performed the opposite experiment by using DX-3 cells characterized by a very low procathepsin L secretion and consequently by a non tumorigenic phenotype [7]. As shown Figure 7D, DX-3 cells were stably transfected with the expression vector encoding for Rab4A wild type form (lane 3) or with the empty myc epitope, used as control (lane 2). Data demonstrated that Rab4A overexpression in transfected DX-3 cells induced at least a 4.7 time increase of procathepsin L secretion (lane 3, lower panel), as compared to DX-3 mock cells (lane 2). In both transfected cells, the expression and the pattern of intracellular cathepsin L forms and of GAPDH expression were not modified (middle panels). Thus, using three distinct methods and three distinct cell lines, we herein demonstrated that both Rab4A expression and Rab4A function regulate procathepsin L secretion in human melanoma cells.

\section{Secretion of unglycosylated procathepsin $\mathrm{L}$ is not modified by Rab4A knock down}

The mannose 6-phosphate receptors (M6PRs) pathway is suspected to be involved in cathepsin L secretion [31]. Thus, we verified the putative involvement of Rab4A in this pathway, by submitting A375SM cells to a $24 \mathrm{~h}$ treatment of tunicamycin, an inhibitor of protein N-glycosylation and consequently of M6PRs pathway. Thus, A375SM cells were transfected with Rab4A siRNAs(1+2) or with control siRNA and also compared to non-transfected cells (Figure 8). First, as expected, in tunicamycin-treated cells (Part B, lanes 4 to 6) the apparent molecular weight of intracellular (upper panel) or secreted (lower panel) procathepsin L was lowered, as compared to the one of cells treated with DMSO alone (Part A, lanes 1 to 3). Second, in tunicamycin-treated cells, while the amount of intracellular procathepsin $L$ and cathepsin $L$ mature forms strongly decreased (upper panel), the amount of secreted procathepsin L increased (lower panel, compare lanes 4 and 5 to lanes 1 and 2). Third, in tunicamycin-treated cells, Rab4A down-regulation still triggered the same $54 \%$ inhibition of procathepsin L secretion (lower panel, lane 6 versus 5), as compared to cells not treated by tunicamycin (lane 3 versus 2 ). Thus, regulation of procathepsin L secretion by Rab4A expression is independent of the glycosylation state of procathepsin $\mathrm{L}$.

\footnotetext{
Regulation of Rab4A expression in vivo switched the phenotype of melanoma cells

We previously demonstrated [7, 14-16] that regulation of procathepsin L secretion switches the phenotype of human melanoma cells. These data and those described above led us to analyze the influence of Rab4A down- or up-regulation on in vivo progression of tumors induced by melanoma cells in nude mice. Thus, we prepared A373SM cells stably transfected with the Rab4AS22N expression vector with the Rab4A dominant negative mutant form (A375SM myc-Rab4AS22N) or with the empty vector (A375SM mock) used as control, as described above in Figure 7A. In parallel, we prepared DX-3 cells stably transfected with the expression vector encoding for Rab4A wild type form (lane 3) or with the empty myc epitope (lane 2) used as control, as described above in Figure 7D. Then, transfected cells were injected s.c. into nude mice. The tumor volumes were monitored on the indicated days and the mean values were reported. As shown Figure 9, A375SM mock cells, transfected with empty vector, induced tumors which attained a mean volume of 510 $\mathrm{mm}^{3}$ on day 38 (curve 1), as did the non-transfected parental cells (not shown). However, when
} 
A375SM cells were stably transfected with the Rab4A dominant negative mutant form (A375SM myc-Rab4AS22N), the size of the tumors strongly decreased by $45 \%$, to a mean volume of 280 $\mathrm{mm}^{3}$ (curve 2). The DX-3 cells stably transfected with empty vector were non- or poorlytumorigenic (curve 3), as the non-transfected parental cells [7]. However, when Rab4A was overexpressed in the DX-3 cells, the phenotype of these cells was modified, i.e. the size of tumors increased to a mean volume of $345 \mathrm{~mm}^{3}$ (curve 4). Thus, down-regulation of Rab4A function or up-regulation of Rab4A expression by triggering the decrease or the increase of procathepsin $\mathrm{L}$ secretion, respectively, switched the tumorigenic phenotype of human melanoma cells. 


\section{DISCUSSION}

We focused on the putative involvement of small Rab GTPase proteins [17] in procathepsin $\mathrm{L}$ secretion in human melanoma cells, by selecting Rab3A, Rab4A, Rab5A, Rab8A, Rab11A, Rab27A and Rab33A involved in distinct regulatory pathways [19, 20-22].

Thus, we herein provide the first demonstration that among these Rab proteins, only Rab4A was clearly involved in the regulation of procathepsin L secretion in human melanoma cells. This new result was supported by complementary data. First, only Rab4A mRNA and protein expression in these four human melanoma cells correlates with their level of procathepsin L secretion, i.e. Ra4A expression is higher in cell lines characterized by a high level of procathepsin $L$ secretion than in cell lines characterized by a low level of procathepsin L secretion. The Rab4B isoform that shares redundant functions with Rab4A [26] was not expressed in these cells. Second, the knock down of Rab4A expression by two specific siRNAs used together, induced a strong $80 \%$ decrease of procathepsin L secretion, without modification of the intracellular expression of cathepsin L protein forms and cathepsin L mRNA. These data ruled out direct off-target effect on cathepsin L mRNA. The use of "off-target" database, (http://rnai.cs.unm.edu/offTarget) with the Rab4A siRNA sequences did not identify any off-targets. The specificity of these two Rab4A siRNAs was supported as they did not modify the expression of GAPDH and other Rab proteins, as Rab3A, Rab5A, Rab8A, Rab11A. The melanoma cells used did not express Rab27A and Rab33A. Rab27A was expressed in some melanoma cell lines [32], while Rab33A was often not expressed in human melanoma cells [22]. Third, Rab4A inhibition by Rab4A siRNAs $(1+2)$ discriminates between the synthesis and the secretion pathways of regulation of procathepsin L. Indeed, such inhibition of Rab4A expression did not modify the pattern and the relative band intensity of the intracellular procathepsin L and cathepsin L mature forms. This is also supported by pulse chase experiments since the turnover of procathepsinL and cathepsin mature forms was not modify in cells transfected with Rab4A siRNAs(1+2), as compared to cells transfected with control siRNA. Fourth, Rab4A involvement in procathepsin L secretion was strengthened by the expression of Rab4A dominant negative mutant form which blocks Rab4A functions: expression of Rab4A dominant negative form inhibits by $58 \%$ procathepsin $\mathrm{L}$ secretion. Fifth, Rab4A over-expression induced a $64 \%$ increase of procathepsin L secretion. Thus, down- or up-regulation of Rab4A expression or Rab4A function triggered decrease or increase of procathepsin L secretion, respectively, without significant modification of the synthesis and maturation processes of intracellular cathepsin L forms.

In addition, the specific involvement of Rab4A in procathepsin L secretion was strongly supported by two different results. First, by analyzing other Rab family members we herein demonstrated that knock down of Rab3A, Rab5A, Rab8A and Rab11A by their specific respective siRNAs, did not modify procathepsin L secretion. Second, down-regulation of Rab4A in human melanoma cells regulated procathepsin L secretion without modifying the secretion of IL-8 and MMP-2. These data are in agreement with others that Rab4A could not be considered as a general regulator of secretion and is involved in only specific secretion pathways, depending on the cell type [19]: Rab4A was involved in the $\mathrm{Ca}^{2+}$-induced exocytosis of a-granules in platelets [33] or in the amylase secretion in pancreatic acinar cells [34]. Furthermore, the absence of secretion of procathepsin B or procathepsin D in these four melanoma cell lines [35 and Frade et al., unpublished data] prevented to analyze the impact of Rab4A expression on these two proenzymes. Interestingly, in Hela cells inhibition of Rab4A by specific siRNA anti-Rab4A did not modify procathepsin D secretion [36].

Furthermore, while the exact mechanism through which Rab4A regulates procathepsin L secretion in human melanoma cells remains unknown, data suggested that further analysis should be conducted at the intracellular level but also at the cell surface level. At the intracellular level, first of all, we demonstrated that Rab4A involvement in the regulation of procathepsin L secretion is not linked to the glycosylation state of procathepsin L, using tunicamycin-treated cells. After being 
initially translated as preprocathepsin $\mathrm{L}$ in the reticulum endoplasmic and transferred through the Golgi, procathepsin L, like other cysteine proteinases, could be translocated to the endosomal compartment. This translocation from the trans-Golgi network (TGN) to the endosomes is mediated by M6PRs and can be inhibited by tunicamycin, the inhibitor of protein $\mathrm{N}$-glycosylation [31]. Thus, our data suggested that procathepsin L may be directed to a Rab4A endosomal compartment through a distinct M6PRs-independent pathway between the TGN and the endosomal compartment. Recently, it was shown that cathepsin D and cathepsin $\mathrm{H}$ could be targeted to lysosome by the sortilin receptor, independently of M6PRs [37]. These authors also suggested that cathepsin L could reach the lysosomes in a M6PRS- and sortilin receptor-independent manner [38]. Second, the fact that Rab11A knock down, at the opposite of Rab4A knock down, had no effect on procathepsin L secretion, should be also taken into account. Indeed, as Rab4A, Rab11A is a regulator of endosomal traffic, i.e. in pathway from endosomes to the plasma membrane [30]. This apparent discrepancy is most likely related to the main localization of Rab4A in early endosomes at the cell periphery [22], which are mainly composed of Rab4A and contain only few amounts of Rab11A, mainly localized in perinuclear recycling endosomes [39]. Third, we could not demonstrate any high or low proteinprotein interaction between Rab4A and procathepsin L (Frade et al., unpublished data), in spite of the two distinct methods used: a) the yeast two-hybrid interaction system; b) coimmunoprecipitation assays of Rab4A and procathepsin L, with covalent cross-linking of intracellular molecules in live cells, while this protocol was successful to identify a new procathepsin L binding protein. Indeed, Maldi-TOF mass spectrometry analysis of cellular components covalent cross-linked to procathepsin L in live cells and isolated by specific immunoprecipitation assays, led us to recently demonstrate that GRP94, one chaperone protein of the endoplasmic reticulum, interacts with procathepsin $\mathrm{L}$ in human melanoma cells. Downregulation of GRP94 expression by specific GRP94 siRNA increased secretion of procathepsin L, whereas the level of intracellular cathepsin L forms was not modified (Frade et al., manuscript in preparation). This new result incites for analysis at the cell surface. Indeed, GRP94 is not only localized in the endoplasmic reticulum, but also expressed at the cell surface, where this chaperone protein was associated to proteases and peptides processing. It was recently demonstrated that, at the cell surface, the chaperone GRP94 is regulating the processing of the metalloprotease ProADAMTS9 [40]. GRP94 was also recently described as involved, at the cell surface, in the crosspresentation of associated peptides [41]. It was previously suggested that GRP94, as other heat shock protein, play important functions at cell surface, in cell signaling and immunity [42]. While, the functional relationship between "heat shock protein" and Rab protein is poorly documented (only 13 citations in PubMed), it was observed by others that heat shoch protein surface 70-positive tumor exosomes are enriched in Rab4 protein [43]. Thus, from all these data we propose the hypothesis that Rab4A may regulate procathepsin L secretion through the chaperone GRP94 protein at the cell-surface level.

In addition, the biological impact of the specific regulation of Rab4A expression and function on the phenotype of human melanoma cells is herein demonstrated. Indeed, down-regulation of Rab4A function in A375SM cells stably transfected with Rab4A dominant negative mutant form decreased by around $50 \%$ the high tumorigenic phenotype of human melanoma cells. Conversely, upregulation of Rab4A expression in DX-3 cells stably transfected with Rab4A wild type form switched their phenotype from non or poorly- to highly-tumorigenic in nude mice. These data provide the molecular basis of a new gene therapy to inhibit the progression of tumors induced by human melanoma cells, as well as we performed with anti-cathepsin L ScFv [15, 16].

In conclusion, we herein provide the first evidence that down-regulation of Rab4A expression and Rab4A function inhibit procathepsin L secretion, without modifications of the expression and the pattern of intracellular procathepsin $L$ and cathepsin $L$ mature forms. By regulating procathepsin $\mathrm{L}$ secretion, Rab4A expression and Rab4A function contribute to the switches of the tumorigenic phenotype of human melanoma cells in nude mice. 


\section{ACKNOWLEDGMENTS}

The experiments on mice were performed with the assistance of Dr. Ming WEI (CellVax Inc., France), at the CERFE of Evry (France).

This work was supported by the INSERM, the Institut National contre le Cancer (INCa), the Association de Recherche contre le Cancer (ARC) and the Ministère de l'Education Nationale et de la Recherche Scientifique (MENRS). 


\section{REFERENCES}

1 Fidler, I. J. (2002) The organ microenvironment and cancer metastasis. Differentiation 70, 489505

2 Frade, R. (1999) Structure and Functions of proteases which cleave human C3 and are expressed on normal or tumor human cells: some are involved in tumorigenic and metastatic properties of human melanoma cells. Immunopharmacology 42, 39-45

3 Frade, R. (1990) Structure and signaling functions of C3 receptors on human B lymphocytes. In: Klaus GB, editor. Transmembrane Signalling in Lymphocytes. Philadelphia, Pa: Saunders Scientific Publishing Co, pp.159-164

4 Frade, R. (1993) Signal transduction through the Epstein-Barr virus receptor in human B lymphocytes. In: d'Alessandro N, Mihich E, Rausa L, Tapiero H, Tritton TR, eds. Cancer Therapy: Differentiation, Immunomodulation and Angiogenesis. Berlin: Nato ASI Series, Springer-Verlag, pp.33-38

5 Lottin-Divoux, S., Jean, D., Le Romancer, M. and Frade, R. (2006) Activation of Epstein-Barr virus/C3d receptor (gp140, CR2, CD21) on human B lymphoma surface triggers Cbl tyrosine phosphorylation, its association with p85 subunit, Crk-L and syk and its dissociation with Vav. Cell Signal 18, 1219-1225

6 Jean, D., Bar-Eli, M., Huang, S., Xie, K., Rodrigues-Lima, F. and Frade, R. (1996) A cysteine proteinase, which cleaves human $\mathrm{C} 3$, the third component of complement, is involved in tumorigenicity and metastasis of human melanoma. Cancer Res. 56, 254-258

7 Frade, R., Rodrigues-Lima, F., Huang, S., Xie, K., Guillaume N. and Bar-Eli, M. (1998) Procathepsin L, a proteinase that cleaves human C3 (the third component of complement), confers high tumorigenic and metastatic properties to human melanoma cells. Cancer Res. 58, 2733-2736

$8 \mathrm{Gal}, \mathrm{S}$. and Gottesman, M.M. (1988) Isolation and sequence of a cDNA for human pro-cathepsin L. Biochem J. 253, 303-306

9 Joseph, L., Chang, L., Stamenkovich, D. and Sukhatme, V.P. (1988) Complete nucleotide and deduced amino acid sequences of human and murine preprocathepsin L. An abundant transcript induced by transformation of fibroblasts. J Clin Invest 81, 1621-1629

10 Turk, B., Turk, D. and Turk, V. (2000) Lysosomal cysteine proteases: more than scavengers. Biochem Biophys Acta 1477, 98-111

11 Troen, B.R., Gal, S. and Gottesmann, M.M. (1987) Sequence and expression of the cDNA for MEP (major excreted protein), a transformation-regulated secreted cathepsin. Biochem J. 246, 731735

12 Amuthan, G., Biswas, G., Ananadatheerthavarada, H.K., Vijayasarathy, C., Shephard, H.M. and Avadhani, N.G. (2002) Mitochondrial stress-induced calcium signaling, phenotypic changes and invasive behavior in human lung carcinoma A549 cells. Oncogene 21, 7839-7849

13 Gocheva, V. and Joyce, J.A. (2007) Cysteine Cathepsins and the cutting edge of cancer invasion. Cell Cycle 6, 60-64

14 Guillaume-Rousselet, N., Jean, D. and Frade, R.(2002) Cloning and characterization of anticathepsin $\mathrm{L}$ single chain variable fragment whose expression inhibits procathepsin $\mathrm{L}$ secretion in human melanoma cells. Biochem J. 367, 219-227

15 Rousselet, N., Mills, L., Jean, D., Tellez, C., Bar-Eli, M. and Frade, R. (2004) Inhibition of tumorigenic and metastatic phenotype of human melanoma cells by anti-cathepsin L single chain variable fragment. Cancer Res. 64,146-151

16 Frade, R., Rousselet, N. and Jean, D. (2008) Intratumoral gene delivery of anti-cathepsin L single-chain variable fragment by lentiviral vector inhibits tumor progression induced by human melanoma cells. Cancer Gene Ther. 15, 591-604

17 Schwartz, S.L., Cao, C., Pylypenko, O., Rak, A. and Wandinger-Ness A. (2007) Rab GTPases at a glance. J. Cell Sci. 120, 3905-10 
18 Ishidoh, K. and Kominami, E. (1998) Gene regulation and extracellular functions of procathepsin L. Biol. Chem. 379: 131-135

19 Fukuda, M. (2008) Regulation of secretory vesicle traffic by Rab small GTPases. Cell Mol Life Sci. 65, 2801-2813

20 Huber, L.A., Pimplikar, S., Parton, R.., Virta, H., Zerial, M. and Simons, K. (1993) Rab8, a small GTPase involved in vesicular traffic between the TGN and the basolateral plasma membrane. J. Cell. Biol. 123, 35-45

21 Maxfield. F.R. and McGraw, T.E. (2004) Endocytic recycling. Nat. Rev. Mol. Cell. Biol. 5, 121132

22 Györffy, B. and Lage, H. (2007) A Web-based data warehouse on gene expression in human malignant melanoma. J. Invest. Dermatol. 127, 394-399

23 Ndong, J., Jean, D., Rousselet, N. and Frade, R. (2009) Down-regulation of the expression of RB18A/MED1, a co-factor of transcription, triggers strong tumorigenic phenotype of human melanoma cells. Int. J. Cancer 124, 2597-2606

24 Jean, D., Rousselet, N. and Frade, R. (2006) Expression of cathepsin L gene in human tumour cells is under the control of distinct regulatory mechanisms. Oncogene 25, 1474-1478

25 Luca, M., Hunt, B., Bucana, C.D., Johnson, J.P., Fidler, I.J and Bar-Eli, M. (1993) Direct Correlation between MUC18 expression and metastatic potential of human melanoma cells. Melanoma Res. 3, 35-41

26 Krawczyk, M., Leimgruber, E., Seguín-Estévez, Q., Dunand-Sauthier, I., Barras, E. and Reith W. (2007) Expression of RAB4B, a protein governing endocytic recycling, is co-regulated with MHC class II genes. Nucleic Acids Res. 35, 595-605

27 Baricos, W.H., Zhou, Y., Mason, R.W. and Barrett, A.J. (1988) Human kidney cathepsins B and L. Characterization and potential role in degradation of glomerular basement membrane. Biochem J. 252, 301-304

28 Rofstad, E.K. and Mathiesen, B. (2010) Metastasis in melanoma xenografts is associated with tumor microvascular density rather than extent of hypoxia. Neoplasia. 11, 889-898

29 VanMeter, T.E., Rooprai, H.K., Kibble, M.M., Fillmore, H.L, Broaddus, W.C. and Pilkington, G.J. (2001) The role of matrix metalloproteinase genes in glioma invasion: co-dependent and interactive proteolysis. J. Neurooncol. 53, 213-235

30 van der Sluijs, P., Hull, M., Webster, P., Mâle, P., Goud, B. and Mellman, I. (1992) The small GTP-binding protein rab4 controls an early sorting event on the endocytic pathway. Cell 70, 729740

31 Luzio, J.P., Pryor, P.R. and Bright, N.A. (2007) Lysosomes: fusion and function. Nat. Rev. Mol. Cell. Biol. 8, 622-632

32 Hoek, K., Rimm, D.L., Williams, K.R., Zhao, H., Ariyan, S., Lin, A., Kluger, H.M., Berger, A.J., Cheng, E., Trombetta, E.S., Wu, T., Niinobe, M., Yoshikawa, K., Hannigan, G.E. and Halaban, R. (2004) Expression profiling reveals novel pathways in the transformation of melanocytes to melanomas. Cancer Res. 64, 5270-5282

33 Shirakawa, R., Yoshioka, A., Horiuchi, H., Nishioka, H., Tabuchi, A. and Kita, T. (2000) Small GTPase Rab4 regulates Ca2+-induced alpha-granule secretion in platelets. J. Biol. Chem. 275, 33844-33849

34 Ohnishi, H., Mine, T., Shibata, H., Ueda, N., Tsuchida, T. and Fujita, T. (1999) Involvement of Rab4 in regulated exocytosis of rat pancreatic acini. Gastroenterology 116, 943-952

35 Jean, D., Rodriguez-Lima, F., Cassinat, B., Hermann, J., Cabane, J. and Frade, R. (1997) Coexpression and secretion of $\mathrm{C} 3$, the third component of complement and a C3-cleaving cysteine proteinase in a highly metastatic human melanoma cell line. Immunol. Let. 58, 107-112

36 Rojas, R., van Vlijmen, T., Mardones, G.A., Prabhu, Y., Rojas, A.L., Mohammed, S., Heck, A.J., Raposo, G., van der Sluijs, P. and Bonifacio, J.S. (2008) Regulation of retromer recruitment to endosomes by sequential action of Rab5 and Rab7. J. Cell. Biol. 183, 513-526 
37 Canuel, M., Korkidakis, A., Konnyu, K. and Morales, C.R. (2008) Sortilin mediates the lysosomal targeting of cathepsins D and H. Biochem. Biophys. Res. Commun. 373, 292-297

38 Canuel, M., Libin, Y. and Morales, C.R. (2009) The interactomics of sortilin: an ancient lysosomal receptor evolving new functions. Histol. Histopathol. 24, 481-492

39 Sönnichsen, B., De Renzis, S., Nielsen, E., Rietdorf, J. and Zerial, M. (2000) Distinct membrane domains on endosomes in the recycling pathway visualized by multicolor imaging of Rab4, Rab5, and Rab11. J. Cell. Biol. 149, 901-914

40 Koo, B.H. and Apte, S.S. (2010) Cell-surface processing of the metalloprotease pro-ADAMTS9 is influenced by the chaperone GRP94/gp96. J. Biol. Chem. 285, 197-205

41 Jockheck-Clark, A.R., Bowers, E.V., Totonchy, M.B., Neubauer, J., Pizzo, S.V. and Nicchitta, C.V. (2010) Re-examination of CD91 function in GRP94 (glycoprotein 96) surface binding, uptake, and peptide cross-presentation. J. Immunol. 185, 6819-30

42 Calderwood, S.K., Mambula, S.S. and Gray, P.J. (2007) Extracellular heat shock proteins in cell signaling and immunity. Ann. N. Y. Acad. Sci. 1113, 28-39

43 Gastpar, R., Gehrmann, M., Bausero, M.A., Asea, A., Gross, C., Schroeder, J.A., Multhoff, G. (2005) Heat shock protein 70 surface-positive tumor exosomes stimulate migratory and cytolytic activity of natural killer cells. Cancer Res. 65, 5238-47 


\section{LEGENDS}

FIGURE 1: Rab protein expression in melanoma cell lines.

Cellular extracts prepared from A375SM (lanes 1), DM-4 (lanes 2), DX-3 (lanes 3), and SB-2 (lanes 4) cells were submitted to SDS-PAGE and analyzed by western blot using specific antiRab3A, Rab8A, Rab4A, Rab5A and Rab11A Abs. GAPDH expression was used as controls. Western blots were quantified, as described in Materials and Methods, using bands of A375SM cells as $100 \%$.

FIGURE 2: Rab mRNA expression in melanoma cell lines.

cDNAs were prepared from A375SM (lanes 1), DM-4 (lanes 2), DX-3 (lanes 3), and SB-2 (lanes 4) cells. Then, Rab mRNA levels were evaluated by semi-quantitative RT-PCR with specific primers for Rab3A, Rab8A, Rab4A, Rab5A and Rab11A. As control, GAPDH was also amplified from the same cDNA.

FIGURE 3: Regulation of procathepsin L secretion by Rab protein knock down.

A375SM cells were non-transfected (NT, lanes 1) or transfected with control siRNAs (lanes 2) or specific siRNAs (lanes 3) for Rab3A (Part A), Rab5A (Part B), Rab4A (Part C), Rab8A (Part D) and Rab11A (Part E). Cellular extracts were prepared $72 \mathrm{~h}$ after transfection and protein expression was analyzed by western blot using specific anti-Rab3A (Part A), Rab5A (Part B), Rab4A (Part C), Rab8A (Part D), Rab11A (Part E), cathepsin L (Parts A-E) and GAPDH (Parts A-E) Abs. Extracellular media, conditioned during $24 \mathrm{~h}$ by A375SM cells, were also analyzed using anticathepsin L Abs (lower gels of Parts A-E). Western blots were quantified as described in Materials and Methods, using data from cells transfected with control siRNA (lane 2) as 100\% (r.b.i. = relative band intensity).

FIGURE 4: Effect of Rab4A knock down on the expression of other Rab proteins. A375SM cells were non-transfected (NT, lanes 1) or transfected with control siRNAs (lanes 2) or with two specific Rab4A siRNAs $(1+2)$ (lanes 3). Rab protein expression was analyzed by western blot $72 \mathrm{~h}$ post transfection using specific anti-Rab3A, Rab8A, Rab4A, Rab5A and Rab11A Abs. Western blots were quantified as described Figure 3.

FIGURE 5: Time course of cathepsin $\mathrm{L}$ biogenesis and maturation in A375SM cells in which Rab4A synthesis was specifically inhibited.

A375SM melanoma cells transfected with control siRNA (C) or Rab4A siRNAs(1+2) (A) were pulsed chased, as described in Materials and methods. Intracellular procathepsin $\mathrm{L}$ and cathepsin $\mathrm{L}$ forms were immunoprecipitated from labeled cell lysates and analyzed under reducing conditions by SDS-PAGE (Upper Part). Each gel was submitted to density analysis and data represented in the graph (Lower Part).

FIGURE 6: Effect of Rab4A knock down on cathepsin L mRNA expression, cathepsin L activity and IL-8 and MMP-2 secretions.

Part A: Rab4A and cathepsin L mRNA expression level was analyzed by semi-quantitative RTPCR on cDNAs prepared from non-transfected A375SM (NT, lane 1), transfected with control siRNA (lane 2) or Rab4A siRNAs(1+2) (lane 3), $72 \mathrm{~h}$ after transfection. GAPDH cDNA, also amplified from the same cDNA, was used as a control for DNA integrity and equal loading. Part B. The cDNA was also analyzed for cathepsin L expression by quantitative Real-Time RT-PCR. Cathepsin L transcript levels were normalized to GAPDH mRNA and are expressed as a percentage of the transcript level measured in non-transfected cells. Part C: Cathepsin L activity was measured using Z-Phe-Arg-AMC in total extracts of non-transfected A375SM cells (lane 1 and 4), cells 
transfected with control siRNA (lane 2) or Rab4A siRNAs(1+2) (lane 3) in the absence (lanes 1, 2 and 3) or presence (lane 4) of the specific inhibitor Z-Phe-Phe-CHN $\mathrm{C}_{2}$ of cathepsin $\mathrm{L}$. In all these samples was added CA-074, the specific cathepsin B Inhibitor III, to rule out any interference with cathepsin B activity. The histogram represents the percentage of relative cathepsin L activity as compared to non-transfected A375SM cells. Part D: secreted IL-8 (black histogram) and secreted MMP-2 (white histogram) were measured by Elisa or western blot respectively, in extracellular media conditioned by A375SM cells non-transfected (NT, lane 1), transfected with control siRNA (lane 2) or Rab4A siRNAs(1+2) (lane 3). The histograms represent the percentages compared to the one of non-transfected A375SM cells. Results are means \pm s.d.

FIGURE 7: Regulation of procathepsin L secretion by Rab4AS22N dominant negative mutant form or by wild type Rab4A overexpression in melanoma cells.

Parts A and B: A375SM parental cells (lanes 1) were stably transfected with either the dominant negative mutant Rab4AS22N expression vector (Part A, lane 3) or with the wild type Rab4A expression vector (Part B, lane 3). In control, cells were transfected with empty vector (lanes 2). Part C: DM-4 melanoma cells non-transfected (NT, lanes 1), transfected with control siRNA (lanes 2) or Rab4A siRNAs(1+2) (lanes 3). Part D: parental DX-3 melanoma cells (lanes 1) were stably transfected with wild type Rab4A expression vector myc-Rab4A (lanes 3) or with myc empty vector (lanes 2), used as control. Then, cellular extracts and extracellular media were prepared and analyzed by western blot using anti-Rab4A, anti-GAPDH and anti-cathepsin L Abs, where needed. In all experiments, western blots were quantified as described in Figure 3, using mock cells as control.

FIGURE 8: Procathepsin L secretion by tunicamycin-treated A375SM cells.

A375SM cells were treated for $24 \mathrm{~h}$ with DMSO alone (lanes 1-3) or tunicamycin in DMSO (lanes 4-6) $48 \mathrm{~h}$ after transfection with siRNAs. Cellular extracts and extracellular media prepared from non-transfected cells (lanes 1 and 4) and from cells transfected with control siRNA (lanes 2 and 5) or Rab4A siRNAs(1+2) (lanes 3 and 6), were analyzed by western blot using anti-cathepsin $L$ and anti-GAPDH Abs. Western blots were quantified as described Figure 3.

FIGURE 9: Effect of Rab4A down-regulation on progression of tumors induced in nude mice by stably transfected A375SM and DX-3 cells.

In a first step, A375SM cells were stably transfected with the dominant negative mutant Rab4AS22N expression vector, as described Figure 7A and DX-3 cells were stably transfected with wild type Rab4A expression vector myc-Rab4A, as described Figure 7D. Then, in all experiments, $7 \times 10^{5}$ A375SM (curves 1 and 2) or DX-3 (curves 3 and 4) cells transfected with respective construct (curves 2 and 4) or empty vector (mock cells) (curves 1 and 3), were injected s.c in nude mice. The tumor progression was monitored by measuring the volume of each mouse's tumor, with calipers, on the indicated days, using six mice per group. $\mathrm{P}$ values were calculated by using the Student's t test and are indicated by a $\star$ when significantly different $(\mathrm{P}$ value $<0.03)$ from control, i. e. curve 2 versus curve 1 and curve 4 versus curve 3 . These curves are representative of three separate sets of experiments. 


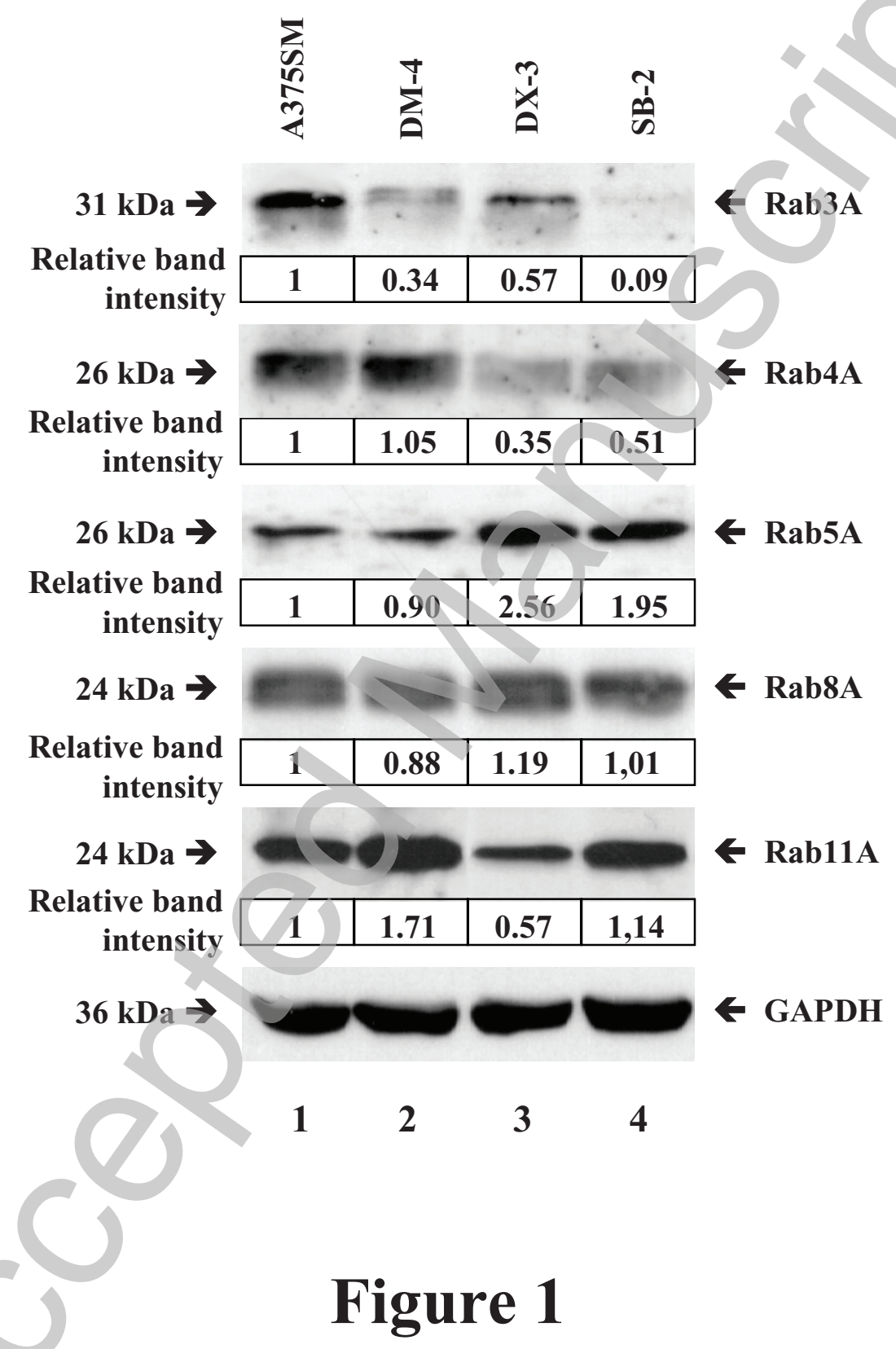


Biochemical Journal Immediate Publication. Published on 18 Apr 2011 as manuscript BJ20110361

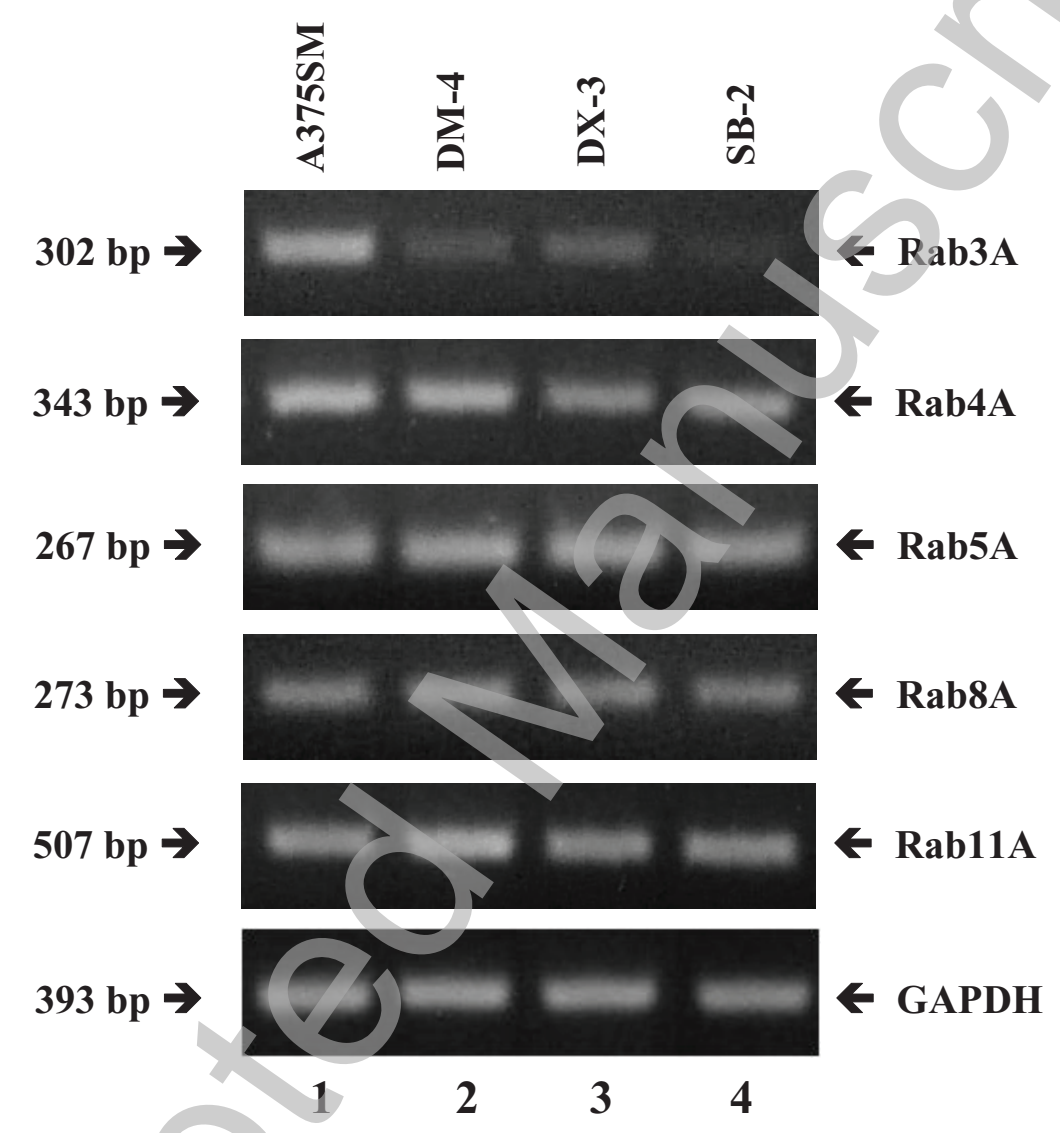

Figure 2 
Biochemical Journal Immediate Publication. Published on 18 Apr 2011 as manuscript BJ20110361

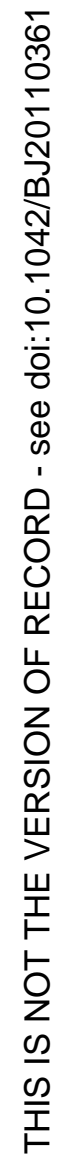

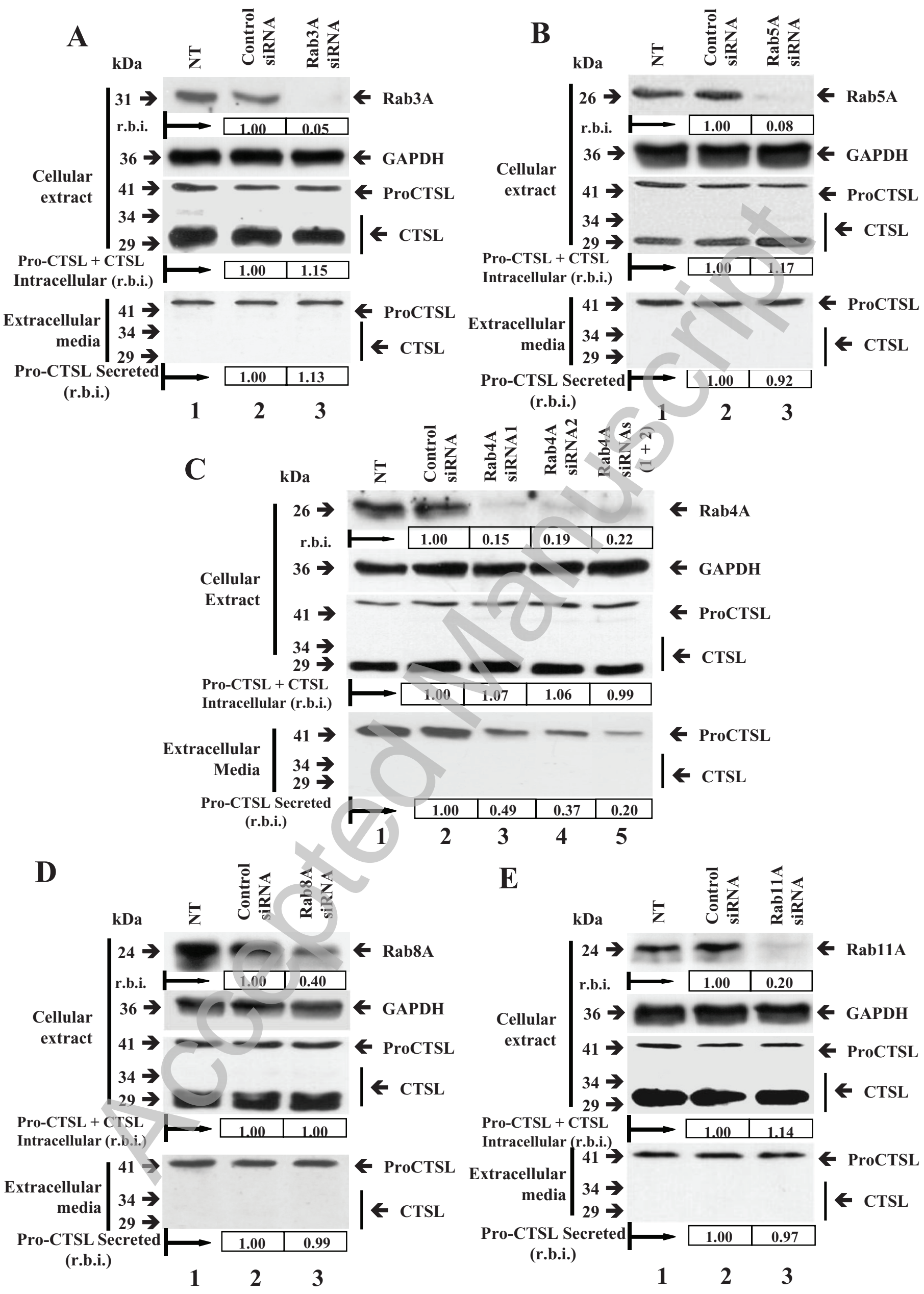

Figure 3

Licenced copy. Copying is not permitted, except with prior permission and as allowed by law.

(C) 2011 The Authors Journal compilation (c) 2011 Portland Press Limited 

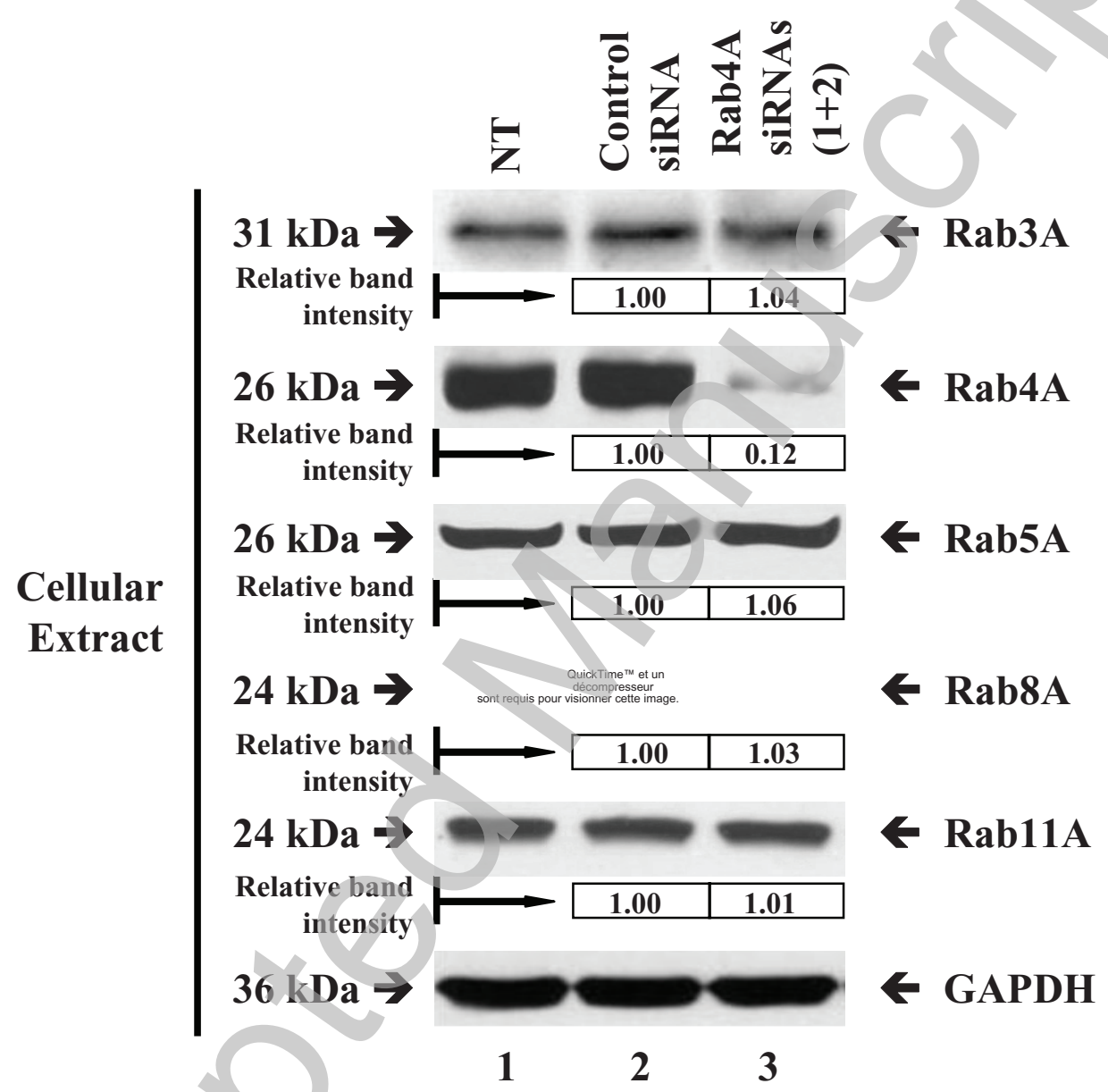

Figure 4 


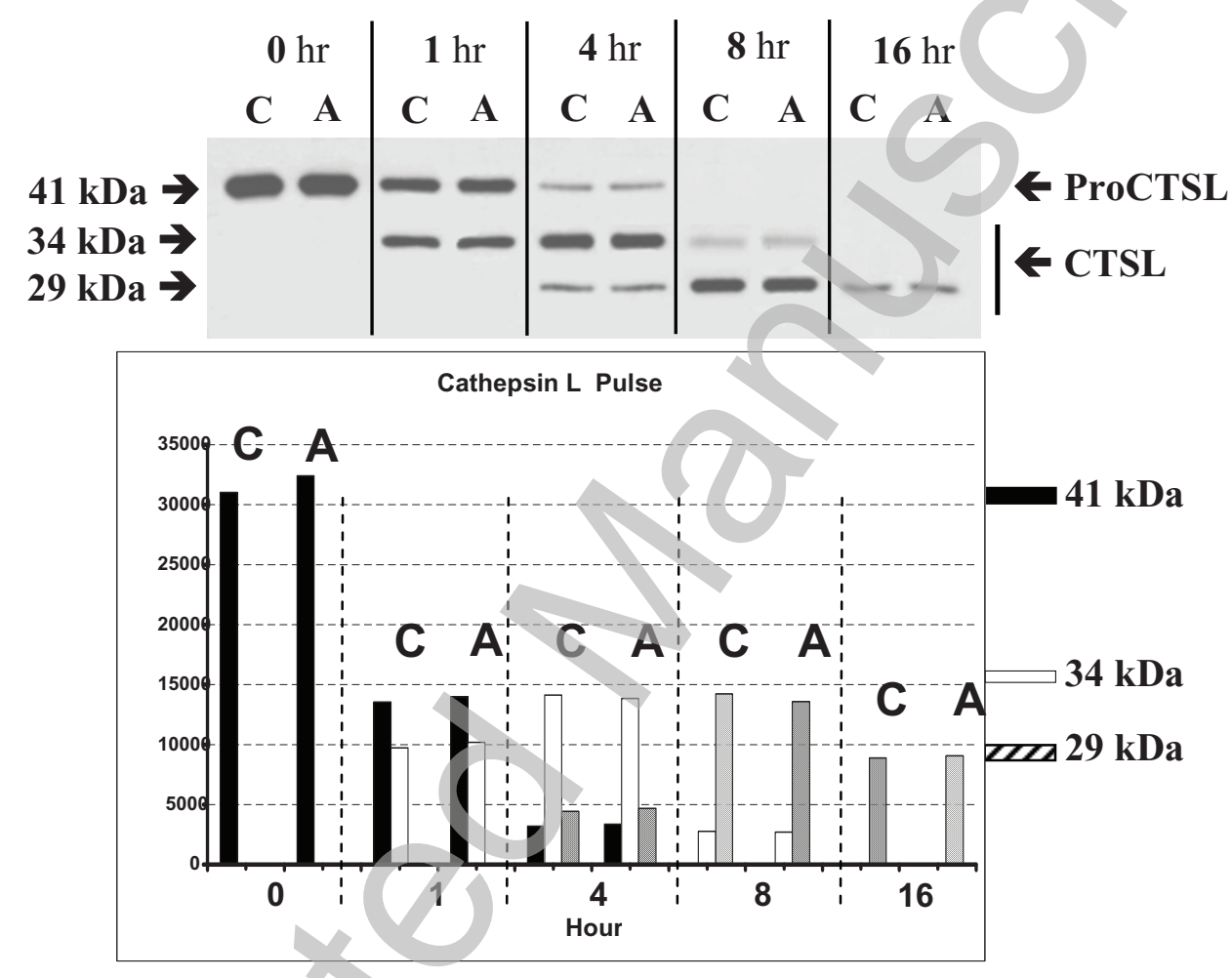

\section{Figure 5}



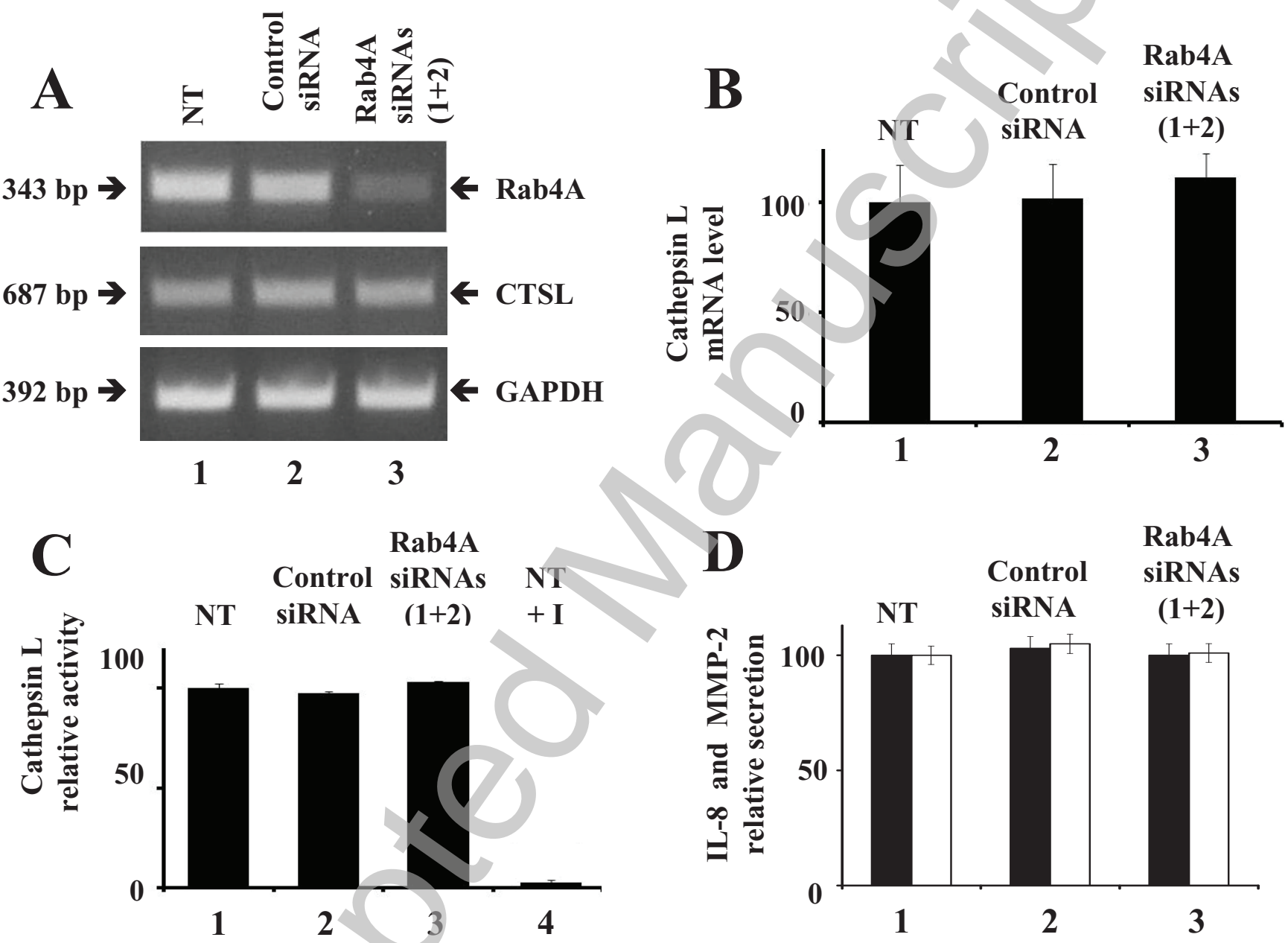

Rab4A

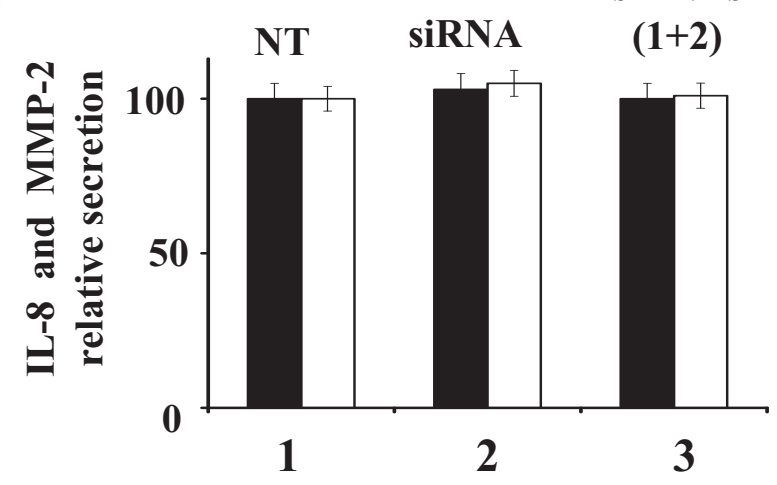

Figure 6 


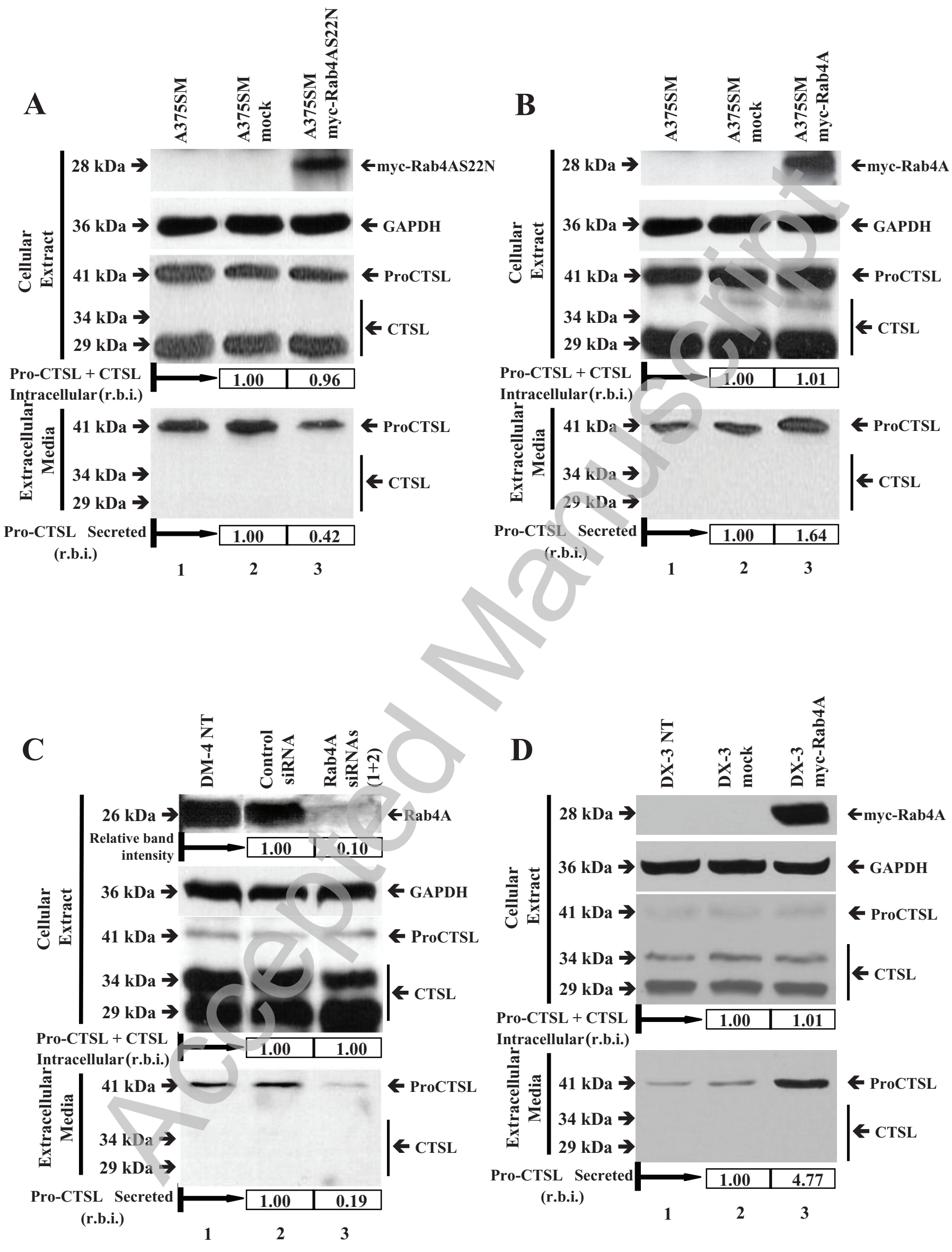

Figure 7

Licenced copy. Copying is not permitted, except with prior permission and as allowed by law. (C) 2011 The Authors Journal compilation (c) 2011 Portland Press Limited 


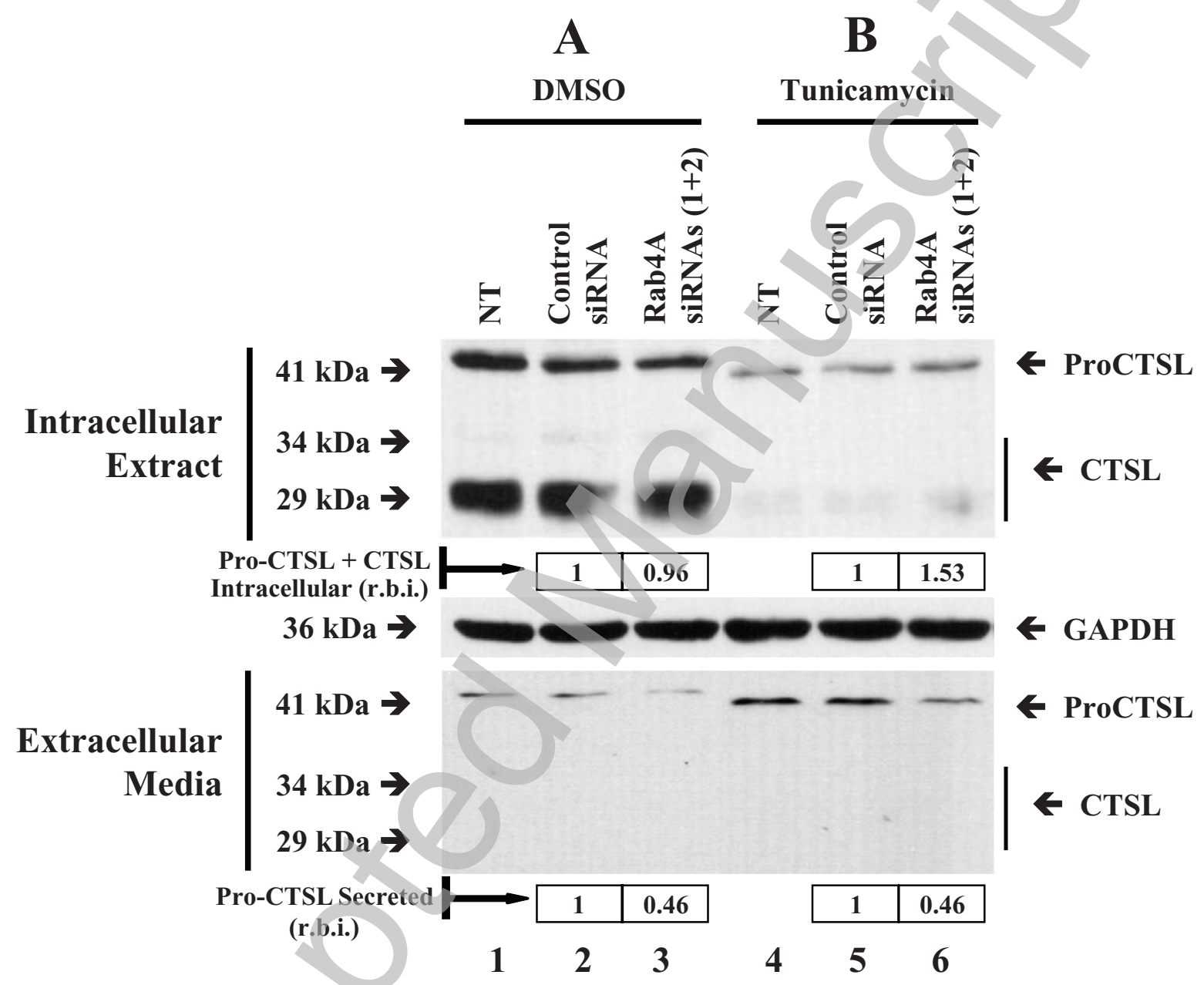

Figure 8 
Biochemical Journal Immediate Publication. Published on 18 Apr 2011 as manuscript BJ20110361

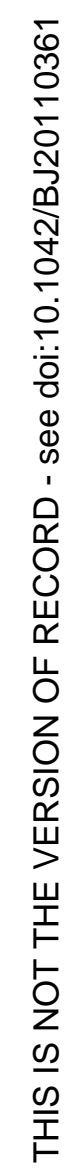

\section{Tumor Growth}

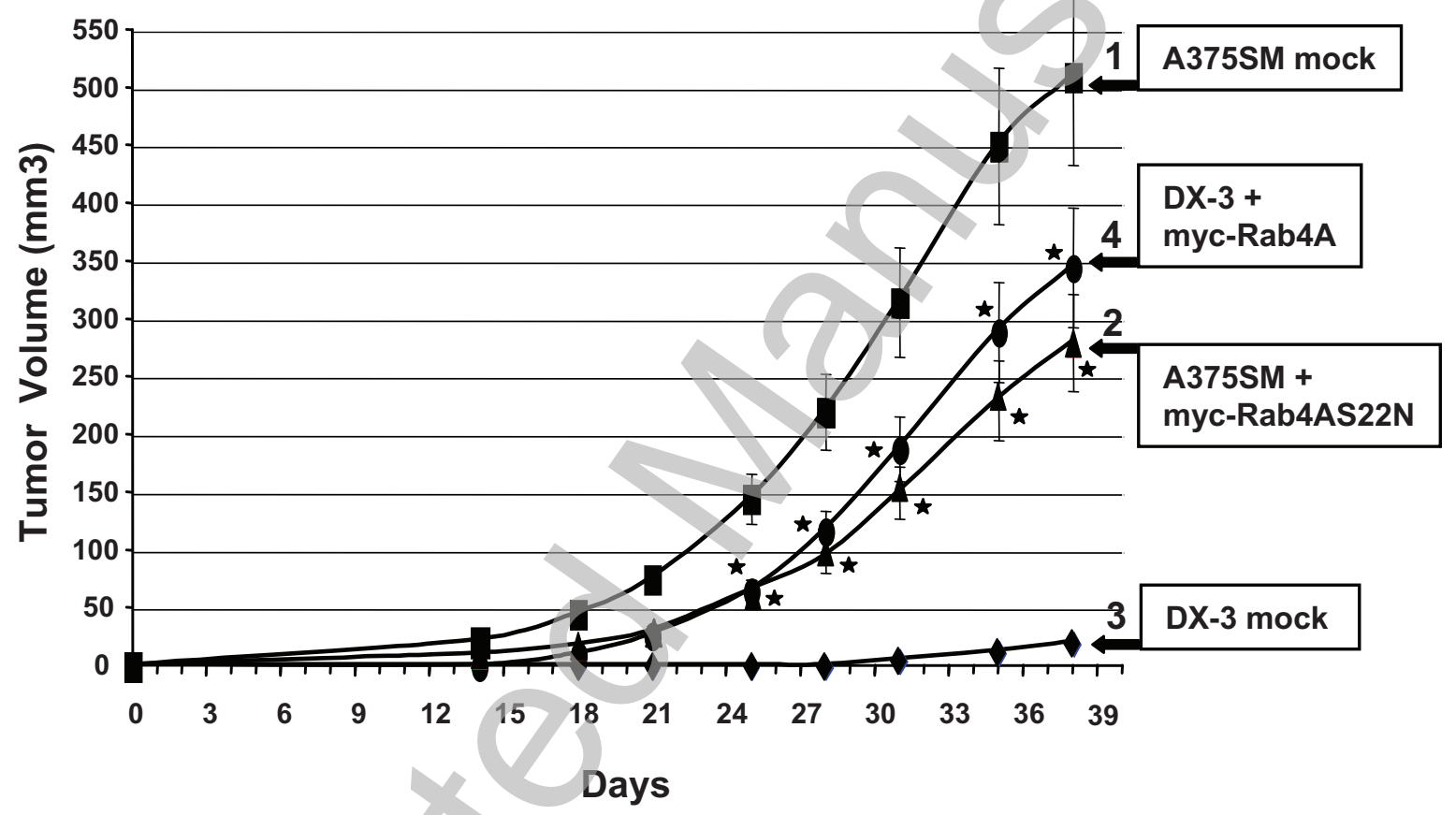

Figure 9

Licenced copy. Copying is not permitted, except with prior permission and as allowed by law.

(C) 2011 The Authors Journal compilation (c) 2011 Portland Press Limited 